\title{
UNIVERSAL COEFFICIENT THEOREM IN TRIANGULATED CATEGORIES
}

\author{
TEIMURAZ PIRASHVILI AND MARÍA JULIA REDONDO
}

\begin{abstract}
We consider a homology theory $h: \mathscr{T} \rightarrow \mathscr{A}$ on a triangulated category $\mathscr{T}$ with values in a graded abelian category $\mathscr{A}$. If the functor $h$ reflects isomorphisms, is full and is such that for any object $x$ in $\mathscr{A}$ there is an object $X$ in $\mathscr{T}$ with an isomorphism between $h(X)$ and $x$, we prove that $\mathscr{A}$ is a hereditary abelian category, all idempotents in $\mathscr{T}$ split and the kernel of $h$ is a square zero ideal which as a bifunctor on $\mathscr{T}$ is isomorphic to $\operatorname{Ext}_{\mathscr{A}}^{1}(h(-)[1], h(-))$.
\end{abstract}

We assume that the reader is familiar with triangulated categories (see [7, 4]). Let us just recall that the triangulated categories were introduced independently by Puppe [6] and by Verdier [7. Following to Puppe we do not assume that the octahedral axiom holds.

If $\mathscr{T}$ is a triangulated category, the shifting of an object $X \in \mathscr{T}$ is denoted by $X[1]$. Assume an abelian category $\mathscr{A}$ is given, which is equipped with an autoequivalence $x \mapsto x[1]$. Objects of $\mathscr{A}$ are denoted by the small letters $x, y, z$, etc, while objects of $\mathscr{T}$ are denoted by the capital letters $X, Y, Z$, etc. A homology theory on $\mathscr{T}$ with values in $\mathscr{A}$ is a functor $h: \mathscr{T} \rightarrow \mathscr{A}$ such that $h$ commutes with shifting (up to an equivalence) and for any distinguished triangle $X \rightarrow Y \rightarrow Z \rightarrow X[1]$ in $\mathscr{T}$ the induced sequence $h(X) \rightarrow h(Y) \rightarrow h(Z)$ is exact. It follows that then one has the following long exact sequence

$$
\cdots \rightarrow h(Z)[-1] \rightarrow h(X) \rightarrow h(Y) \rightarrow h(Z) \rightarrow h(X)[1] \rightarrow \cdots
$$

In what follows $\operatorname{Ext}_{\mathscr{A}}^{1}(x, y)$ denotes the equivalence classes of extensions of $x$ by $y$ in the category $\mathscr{A}$ and we assume that these classes form a set.

In this paper we prove the following result:

Theorem 1. Let $h: \mathscr{T} \rightarrow \mathscr{A}$ be a homology theory. Assume the following conditions hold

i) $h$ reflects isomorphisms,

ii) $h$ is full.

Then the ideal

$$
\mathbb{I}=\left\{f \in \operatorname{Hom}_{\mathscr{T}}(X, Y) \mid h(f)=0\right\}
$$

is a square zero ideal. Suppose additionally the following condition holds

iii) for any short exact sequence $0 \rightarrow x \rightarrow y \rightarrow z \rightarrow 0$ in $\mathscr{A}$ with $x \cong h(X)$ and $z \cong h(Z)$ there is an object $Y \in \mathscr{T}$ and an isomorphism $h(Y) \cong y$ in $\mathscr{A}$.

2000 Mathematics Subject Classification. 18E30.

The second author is a researcher from CONICET, Argentina. 
Then $\mathbb{I}$ is isomorphic as a bifunctor on $\mathscr{T}$ to

$$
(X, Y) \mapsto \operatorname{Ext}_{\mathscr{A}}^{1}(h(X)[1], h(Y)) .
$$

In particular for any $X, Y \in \mathscr{T}$ one has the following short exact sequence

$$
0 \rightarrow \operatorname{Ext}_{\mathscr{A}}^{1}(h(X)[1], h(Y)) \rightarrow \mathscr{T}(X, Y) \rightarrow \operatorname{Hom}_{\mathscr{A}}(h(X), h(Y)) \rightarrow 0 .
$$

Moreover, if we replace condition (iii) by the stronger condition

iv) for any object $x \in \mathscr{A}$ there is an object $X \in \mathscr{T}$ and an isomorphism $h(X) \cong x$ in $\mathscr{A}$,

then $\mathscr{A}$ is a hereditary abelian category and all idempotents in $\mathscr{T}$ split.

Thus this is a sort of "universal coefficient theorem" in triangulated categories.

Our result is a one step generalization of a well-known result which claims that if $h$ is an equivalence of categories then $\mathscr{A}$ is semi-simple meaning that $\operatorname{Ext}_{\mathscr{A}}^{1}=0$ (see for example [4 p. 250]). As was pointed out by J. Daniel Christensen our theorem generalizes Theorem 1.2 and Theorem 1.3 of [3] on phantom maps. Indeed let $\mathscr{S}$ be the homotopy category of spectra or, more generally, a triangulated category satisfying axioms 2.1 of 3 and let $\mathscr{A}$ be the category of additive functors from finite objects of $\mathscr{S}$ to the category of abelian groups. The category $\mathscr{A}$ has a shifting, which is given by $(F[1])(X)=F(X[1]), F \in \mathscr{A}$. Moreover let $h: \mathscr{S} \rightarrow \mathscr{A}$ be a functor given by $h(X)=\pi_{0}(X \wedge(-))$. Then $h$ is a homology theory for which the assertions i)-iii) hold and $\mathbb{I}(X, Y)$ consists of phantom maps from $X$ to $Y$. Hence by the first part of theorem we obtain the familiar properties of phantom maps.

Before we give a proof of the Theorem, let us explain notations involved on it. The functor $h$ reflects isomorphisms, this means that $f \in \operatorname{Hom}_{\mathscr{T}}(X, Y)$ is an isomorphism provided $h(f)$ is an isomorphism in $\mathscr{A}$. This holds if and only if $X=0$ as soon as $h(X)=0$. Moreover $h$ is full, this means that the homomorphism $\mathscr{T}(X, Y) \rightarrow \operatorname{Hom}_{\mathscr{A}}(h(X), h(Y))$ given by $f \mapsto h(f)$ is surjective for all $X, Y \in$ $\mathscr{T}$. Furthermore an abelian category $\mathscr{A}$ is hereditary provided for any two-fold extension

$$
0 \longrightarrow u \stackrel{\hat{\alpha}}{\longrightarrow} v \stackrel{\hat{\beta}}{\longrightarrow} w \stackrel{\hat{\gamma}}{\longrightarrow} x \longrightarrow 0
$$

there exists a commutative diagram with exact rows

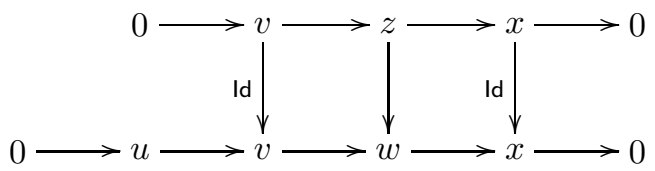

This exactly means that $\mathrm{Ext}_{\mathscr{A}}^{2}=0$, where Ext is understood a lá Yoneda. Let us also recall that an ideal $\mathbb{I}$ in an additive category $\mathbb{A}$ is a sub-bifunctor of the bifunctor $\operatorname{Hom}_{\mathbb{A}}(-,-): \mathbb{A}^{o p} \times \mathbb{A} \rightarrow \mathbb{A} b$. It follows that $\mathbb{I}$ is an additive bifunctor. One can form the quotient category $\mathbb{A} / \mathbb{I}$ in an obvious way, which is an additive category. One says that $\mathbb{I}^{2}=0$ provided $g f=0$ as soon as $f \in \mathbb{I}(A, B)$ and $g \in \mathbb{I}(B, C)$. In this case the bifunctor $\mathbb{I}: \mathbb{A}^{o p} \times \mathbb{A} \rightarrow \mathbb{A} b$ factors through the quotient category $\mathbb{A} / \mathbb{I}$ in a unique way. 
Proof. It is done in several steps.

First step. The equality $\mathbb{I}^{2}=0$. To make notations easier we denote $h(X), h(Y)$ simply by $x, y$, etc. Moreover, for a morphism $\alpha: X \rightarrow Y$, we let $\hat{\alpha}: x \rightarrow y$ be the morphism $h(\alpha)$. Suppose $\alpha: X \rightarrow Y$ and $\beta: Y \rightarrow Z$ are morphisms such that $\hat{\alpha}=0$ and $\hat{\beta}=0$. We have to prove that $\gamma:=\beta \alpha$ is the zero morphism. By the morphisms axiom there is a diagram of distinguished triangles

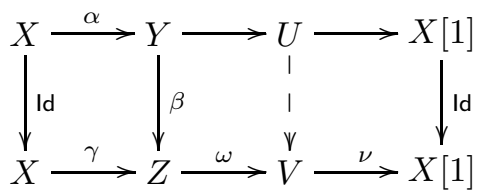

Apply $h$ to get a commutative diagram with exact rows

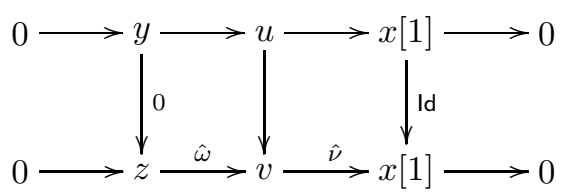

It follows that there is a morphism $\hat{\mu}: x[1] \rightarrow v$ in $\mathscr{A}$ such that $\hat{\nu} \hat{\mu}=\operatorname{Id}_{x[1]}$. Thus $(\hat{\omega}, \hat{\mu}): z \oplus x[1] \rightarrow v$ is an isomorphism. Since $h$ is full, we can find $\mu: X[1] \rightarrow V$ which realizes $\hat{\mu}$, meaning that $h(\mu)=\hat{\mu}$. The morphism $(\omega, \mu): Z \oplus X[1] \rightarrow V$ is an isomorphism, because $h$ reflects isomorphisms. In particular $\omega$ is a monomorphism and therefore $\gamma=0$ and first step is done.

For objects $X, Y \in \mathscr{T}$ we put

$$
\mathbb{I}(X, Y):=\left\{\alpha \in \operatorname{Hom}_{\mathscr{A}}(X, Y) \mid h(\alpha)=0\right\} .
$$

We have just proved that $\mathbb{I}^{2}=0$. In particular $\mathbb{I}$ as a bifunctor factors through the category $\mathscr{T} / \mathbb{I}$. The next step shows that it indeed factors through the category $\mathscr{A}$ and a quite explicit description of this bifunctor is given.

Second step. Bifunctorial isomorphism $\mathbb{I}(X, Y) \cong \operatorname{Ext}_{\mathscr{A}}^{1}(h(X)[1], h(Y))$. We put as usual $x=h(X), y=h(Y)$, etc. Let $\alpha: X \rightarrow Y$ be an element of $\mathbb{I}(X, Y)$. Consider a distinguished triangle

$$
X \stackrel{\alpha}{\rightarrow} Y \stackrel{\beta}{\rightarrow} Z \stackrel{\gamma}{\rightarrow} X[1]
$$

By applying $h$ one obtains the following short exact sequence

$$
0 \rightarrow y \stackrel{\hat{\beta}}{\rightarrow} z \stackrel{\hat{\gamma}}{\rightarrow} x[1] \rightarrow 0
$$

whose class in $\operatorname{Ext}_{\mathscr{A}}^{1}(x[1], y)$ is independent on the choice of the triangle in (2) and it is denoted by $\Xi(\alpha)$. In this way one obtains the binatural transformation $\Xi: \mathbb{I} \rightarrow \operatorname{Ext}_{\mathscr{A}}^{1}((-)[1],(-))$. We claim that $\Xi$ is an isomorphism. Indeed, if $\Xi(\alpha)=0$, then there exists a section $\hat{\mu}: x[1] \rightarrow z$ of $\hat{\gamma}$ in (3). Then $(\hat{\beta}, \hat{\mu}): y \oplus x[1] \rightarrow z$ is an isomorphism. Since $h$ is full, we can find $\mu: X[1] \rightarrow Z$ which realizes $\hat{\mu}$. The morphism $(\beta, \mu): Y \oplus X[1] \rightarrow Z$ is an isomorphism, because $h$ reflects isomorphisms. In particular $\beta$ is a monomorphism and therefore $\alpha=0$. Hence $\Xi$ is a monomorphism. Let us take any element in $\operatorname{Ext}_{\mathscr{A}}^{1}(x[1], y)$, which is represented by a short exact sequence, say the sequence (3). Take any realization $\beta: Y \rightarrow Z$ of $\hat{\beta}$. By Lemma $\square$ below we obtain the following distinguished triangle

$$
X \stackrel{\alpha}{\rightarrow} Y \stackrel{\beta}{\rightarrow} Z \stackrel{\gamma}{\rightarrow} X[1]
$$


containing $\beta$. It follows that $\Xi(\alpha)$ represents our original element in $\operatorname{Ext}_{\mathscr{A}}^{1}(x[1], y)$. Hence $\Xi$ is an isomorphism.

Third step. $\mathscr{A}$ is hereditary. Let (11) be a two-fold extension in $\mathscr{A}$. We put $y=\operatorname{Im}(\hat{\alpha})$. Thus the exact sequence (1) splits in the following two short exact sequences

$$
0 \rightarrow u \stackrel{\hat{\alpha}}{\rightarrow} v \stackrel{\hat{\mu}}{\rightarrow} y \rightarrow 0
$$

and

$$
0 \rightarrow y \stackrel{\hat{\nu}}{\rightarrow} w \stackrel{\hat{\gamma}}{\rightarrow} x \rightarrow 0
$$

with $\hat{\beta}=\hat{\nu} \hat{\mu}$. Using Assumption iii) and without loss of generality we can assume that $u, v, w, x$ as well as $\hat{\alpha}$ and $\hat{\gamma}$ have realizations. By Lemma 1 below we obtain the following distinguished triangles

$$
U \stackrel{\alpha}{\rightarrow} V \stackrel{\mu}{\rightarrow} Y \stackrel{\xi}{\rightarrow} U[1]
$$

and

$$
Y \stackrel{\nu}{\rightarrow} W \stackrel{\gamma}{\rightarrow} X \stackrel{\chi}{\rightarrow} Y[1] .
$$

Since $\hat{\mu}$ is an epimorphism and $\hat{\nu}$ is a monomorphism it follows that $h(\xi)=0$ and $h(\chi)=0$. Thus $\xi \circ \chi[-1]=0$ thanks to the fact that $\mathbb{I}^{2}=0$. Therefore there exists $\lambda: X[-1] \rightarrow V$ such that $\mu \circ \lambda=\chi[-1]$, in other words one has the following commutative diagram

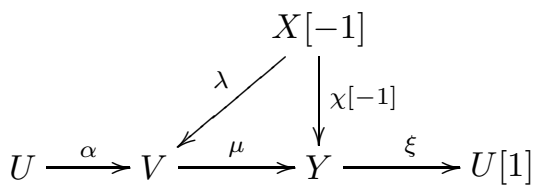

We claim that one can always find $\lambda$ with property $h(\lambda)=0$. Indeed, for a given $\lambda$ with $\mu \circ \lambda=\chi[-1]$ one obtains the following diagram after applying $h$ :

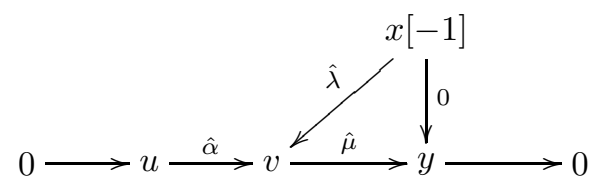

Thus $\hat{\lambda}=\hat{\alpha} \circ \hat{\phi}$, for some $\phi: X[-1] \rightarrow U$. Now it is clear that $\lambda^{\prime}=\lambda-\alpha \circ \phi$ has the expected properties $h\left(\lambda^{\prime}\right)=0$ and $\mu \circ \lambda^{\prime}=\chi[-1]$, and the claim is proved.

One can use the morphisms axiom to conclude that there exists a commutative diagram

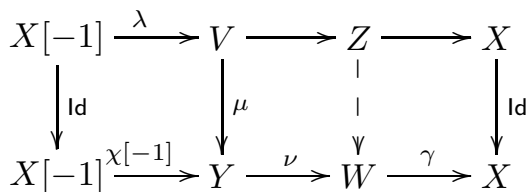

Since $h(\lambda)=0$, by applying $h$ one obtains the following commutative diagram

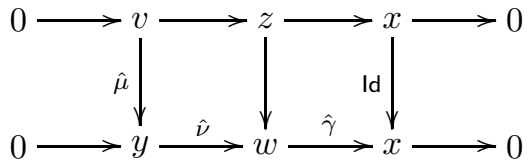


which shows that one has a commutative diagram with exact rows

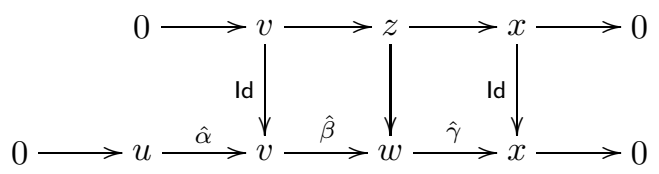

Thus $\mathscr{A}$ is hereditary.

Forth step. Idempotents split in $\mathscr{T}$. Let $\operatorname{Idem}(\mathscr{T})$ be the idempotent completion of $\mathscr{T}$ (see [5] or [1]). We have to show that the canonical functor $\mathscr{T} \rightarrow \operatorname{Idem}(\mathscr{T})$ is an equivalence of categories. One can summarize the previous steps saying that the category $\mathscr{T}$ is a linear extension of $\mathscr{A}$ by the bifunctor $(X, Y) \mapsto \operatorname{Ext}_{\mathscr{A}}^{1}(h(X)[1], h(Y))$ in the sense of Baues and Wirsching [2]. Now one can use Proposition 3.2 of [5] to conclude that $\mathscr{T} \rightarrow \operatorname{Idem}(\mathscr{T})$ is indeed an equivalence of categories.

An alternative proof can be done using the result of [1] and Corollary 2 below which uses only the first three steps. Indeed, by [1, the category $\mathscr{T}^{\prime}=\operatorname{Idem}(\mathscr{T})$ carries a natural triangulated structure. Since $\mathscr{A}$ is an abelian category, all idempotents in $\mathscr{A}$ split and it follows from the universal property of the idempotent completion that the functor $h$ has a unique extension $\mathscr{T}^{\prime} \rightarrow \mathscr{A}$, which is denoted by $h^{\prime}$. We claim that the functor $h^{\prime}$ reflects isomorphisms. Indeed, if $X^{\prime}$ is an object in $\mathscr{T}$ such that $h^{\prime}\left(X^{\prime}\right)=0$, then there exits an object $Y^{\prime}$ such that $Z=X^{\prime} \oplus Y^{\prime}$ lies in $\mathscr{T}$. Let $e: Z \rightarrow Z$ be given by $e(x, y)=(0, y)$. Then $h(Z)=h^{\prime}\left(Y^{\prime}\right)$ and therefore $h(e)$ is an isomorphism. By our assumption on $h$ it follows that $e$ is an isomorphism and hence $X^{\prime}=0$. It is clear that $h^{\prime}$ is full and realizes all objects of $\mathscr{A}$. Hence the conditions of Corollary 2 below hold and therefore $\mathscr{T} \rightarrow \operatorname{Idem}(\mathscr{T})$ is an equivalence of categories.

Lemma 1. Let $h: \mathscr{T} \rightarrow \mathscr{A}$ be a homology theory. Assume $h$ reflects isomorphisms and is full. Suppose there is given a morphism $\alpha: U \rightarrow V$, an object $W$ in $\mathscr{T}$ and a short exact sequence

$$
0 \rightarrow u \stackrel{\hat{\alpha}}{\rightarrow} v \stackrel{\hat{\beta}}{\rightarrow} w \rightarrow 0
$$

in $\mathscr{A}$, where as usual $u=h(U), v=h(V), w=h(W)$ and $\hat{\alpha}=h(\alpha)$. Then there exists a distinguished triangle

$$
U \stackrel{\alpha}{\rightarrow} V \stackrel{\beta}{\rightarrow} W \stackrel{\gamma}{\rightarrow} U[1]
$$

such that $h(\beta)=\hat{\beta}$. The dual statement is also true: Suppose there is given a morphism $\beta: V \rightarrow W$, an object $U$ in $\mathscr{T}$ and a short exact sequence

$$
0 \rightarrow u \stackrel{\hat{\alpha}}{\rightarrow} v \stackrel{\hat{\beta}}{\rightarrow} w \rightarrow 0
$$

in $\mathscr{A}$, where $\hat{\beta}=h(\beta)$. Then there exists a distinguished triangle

$$
U \stackrel{\alpha}{\rightarrow} V \stackrel{\beta}{\rightarrow} W \stackrel{\gamma}{\rightarrow} U[1]
$$

such that $h(\alpha)=\hat{\alpha}$.

Proof. Take any distinguished triangle containing $\alpha$,

$$
U \stackrel{\alpha}{\rightarrow} V \stackrel{\eta}{\rightarrow} Z \stackrel{\epsilon}{\rightarrow} U[1] .
$$

Apply $h$ to get a short exact sequence

$$
0 \rightarrow u \stackrel{\hat{\alpha}}{\rightarrow} v \stackrel{\hat{\eta}}{\rightarrow} z \rightarrow 0 .
$$


Then we get the following commutative diagram

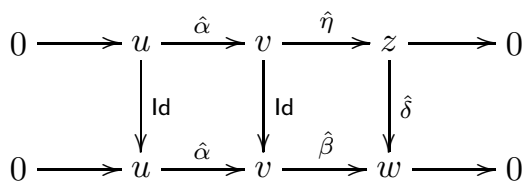

with $\hat{\delta}$ an isomorphism. By assumption one can realize $\hat{\delta}$ to obtain an isomorphism $\delta: Z \rightarrow W, h(\delta)=\hat{\delta}$. Then we have an isomorphism of triangles

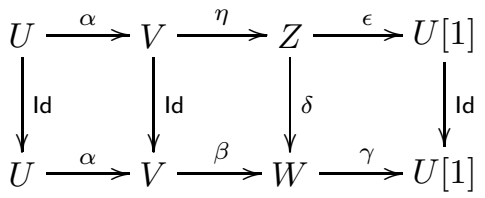

where $\beta=\delta \eta$ and $\gamma=\epsilon \circ \delta^{-1}$. It follows that the triangle

$$
U \stackrel{\alpha}{\rightarrow} V \stackrel{\beta}{\rightarrow} W \stackrel{\gamma}{\rightarrow} U[1]
$$

is also a distinguished triangle. Thus the first statement is proved. The dual argument gives the second result.

Corollary 2. Let $j: \mathscr{T} \rightarrow \mathscr{T}^{\prime}$ be a triangulated functor between triangulated categories. Assume $h^{\prime}: \mathscr{T}^{\prime} \rightarrow \mathscr{A}$ is a homological functor satisfying the conditions i), ii) and iv) of Theorem 1] If the homology functor $h=h^{\prime} \circ j: \mathscr{T} \rightarrow \mathscr{A}$ also satisfies the same conditions then $j$ is an equivalence of categories.

Proof. First observe that the functor $j$ is full and faithful because for any pair of objects $X, Y \in \mathscr{T}$ both abelian groups $\mathscr{T}(X, Y)$ and $\mathscr{T}^{\prime}(j X, j Y)$ are part of the equivalent extensions of $\operatorname{Hom}_{\mathscr{A}}(h(X), h(Y))$ by $\operatorname{Ext}_{\mathscr{A}}^{1}(h(X)[1], h(Y))$. If now $X^{\prime}$ is an object in $\mathscr{T}^{\prime}$ then there is an object $X$ in $\mathscr{T}$ and an isomorphism $\hat{\alpha}: h(X) \rightarrow$ $h^{\prime}\left(X^{\prime}\right)$ in $\mathscr{A}$. But $h(X)=h^{\prime}(j(X))$ and $h^{\prime}$ is full so $\hat{\alpha}=h^{\prime}(\alpha)$ for a morphism $\alpha: j X \rightarrow X^{\prime}$, which is an isomorphism because $h^{\prime}$ reflects isomorphisms.

ACKNOWLEDGEMENTS. The first author was supported by the University of Bielefeld and C.N.R.S. He also acknowledge discussions with Vincent Franjou, Bernhard Keller, Claus Michael Ringel and Stefan Schwede.

\section{REFERENCES}

[1] P. Balmer and M. Schlichting. Idempotent completion of triangulated categories. J. of Algebra, 236 (2001) , 819-834.

[2] H.-J. Baues and G. Wirsching. Cohomology of small categories. J. Pure Appl. Algebra 38 (1985), 187-211.

[3] J.D. Christensen and N. P. Strickland. Phantom maps and homology theories. Topology 37 (1998), no. 2, 339-364.

[4] S. I. Gelfand and Y. I. Manin. Methods of homological algebra. Second edition. Springer Monographs in Mathematics. Springer-Verlag, Berlin, 2003. xx+372 pp.

[5] T. Pirashvili. Projectives are free for nilpotent algebraic theories. Algebraic $K$-theory and its applications (Trieste, 1997), 589-599, World Sci. Publishing, River Edge, NJ, 1999.

[6] D. Puppe. On the structure of stable homotopy theory. Colloquium on algebraic topology. Aarhus Universitet Matematisk Institut (1962), 65-71. 
[7] J. L. Verdier. Des Catégories derivées. des catégories abéliennes. Astérisque v. 239 (1996). $253 \mathrm{pp}$

Department of Mathematics, University of Leicester, Leicester LE1 7RH, United KinGDOM.

Instituto de Matemática, Universidad Nacional del Sur, Av. Alem 1253, (8000) Bahía Blanca, Argentina. 